\title{
Pflege in Zeiten von Corona: Ergebnisse einer deutschlandweiten Querschnittbefragung von ambulanten Pflegediensten und teilstationären Einrichtungen
}

\author{
Karin Wolf-Ostermann ${ }^{1}$, Annika Schmidt¹ , Benedikt Preuß², \\ Franziska Heinze ${ }^{2}$, Kathrin Seibert ${ }^{1}$, Anna-Carina Friedrich ${ }^{1}$, \\ Dominik Domhoff ${ }^{1}$, Claudia Stolle ${ }^{1}$, Heinz Rothgang ${ }^{2}$ \\ IInstitut für Public Health und Pflegeforschung (IPP), Universität Bremen \\ ${ }^{2}$ SOCIUM Forschungszentrum Ungleichheit und Sozialpolitik, Universität Bremen
}

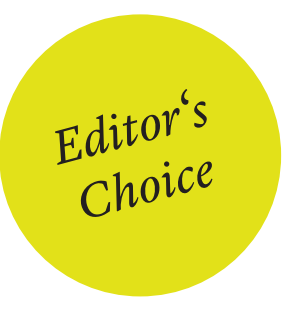

\begin{abstract}
Zusammenfassung: Hintergrund: Pflegebedürftige sind aufgrund des Alters, bestehenden Vorerkrankungen und der Schwere von Krankheitsverläufen von der COVID-19-Pandemie besonders betroffen. Eine Vielzahl täglicher direkter Kontakte von Pflegekräften und Pflegebedürftigen erhöhen die Gefahr für eine Übertragung der Erkrankung. Ziel: Die Studie zielt darauf ab, (I) Fallzahlen ambulanter Pflegedienste und teilstationärer Pflegeeinrichtungen in Zusammenhang mit COVID-19, (II) personelle und materielle Ressourcen, (III) die Versorgungssituation von Pflegebedürftigen und (IV) den organisatorischen Umgang mit der Pandemiesituation zu beschreiben sowie Unterstützungswünsche an die Politik zu skizzieren. Methoden: Mehr als 12000 ambulante und teilstationäre Pflegeeinrichtungen wurden zur Teilnahme an einer Online-Befragung eingeladen (Gelegenheitsstichprobe), 701 Pflegedienste (Rücklauf 7,3\%) und 96 teilstationäre Einrichtungen (Rücklauf 3,5\%) nahmen teil. Ergebnisse: Fast ein Drittel der befragten Pflegedienste (30,1\%, 189/627) sind durch (Verdachts-)Fälle von COVID-19 bei Klient_innen betroffen. Jeweils etwa die Hälfte der Dienste berichten von einer verringerten Inanspruchnahme von SGB $V$ (47,6\%, 288/605) bzw. SGB XI-Leistungen (59,7\%, 375/628). Zwei Drittel der teilstationären Einrichtungen (65,6\%, 63/96) geben an, aufgrund der Pandemie derzeit geschlossen zu sein. Ebenso berichteten fast die Hälfte der Dienste (45,8\%, 253/552), dass die Versorgung bei verringerter Leistungsinanspruchnahme von ambulanten Leistungen gefährdet/instabil oder sogar nicht sichergestellt sei. Schlussfolgerung: Die Ergebnisse zeigen, dass ambulant versorgten Pflegebedürftigen in der vorliegenden Pandemie - und auch mit Blick auf eine mögliche zweite Welle - eine erhöhte Aufmerksamkeit in Bezug auf prekäre Versorgungssituationen zukommen sollte.
\end{abstract}

Schlüsselwörter: Ambulante Pflege; COVID-19; Langzeitpflege, Schutzmaterialien; Personalsituation

\section{Care in times of Corona: Results of a cross-sectional study in German home care services}

\begin{abstract}
Background: People in need of care are particularly affected by the COVID-19 pandemic due to their age and previous illnesses. At the same time, a large number of daily contacts between nursing staff and those in need of care increase the risk of transmission of the disease. Aim: The study aims to illustrate (I) to what extent home care services and semi-residential care facilities are affected by COVID-19, (II) human and material resources, (III) the care situation of those in need of care and (IV) the organizational handling of the situation and outline support requests to politicians. Methods: More than 12,000 home-care nursing services and semi-residential care facilities were invited to participate in an online survey with quantitative and qualitative elements, 701 nursing services (response rate 7,3\%) and 96 semi-residential facilities (response rate 3,5\%) took part. Results: Almost a third $(30,1 \%, 189 / 627)$ of the nursing services surveyed are affected by confirmed or suspected cases of COVID-19 among clients. About half of the services report a reduced use of SGB V (47,6\%, 288/605) or SGB XI services (59,7\%, $375 / 628)$. Two thirds of the semi-residential facilities $(65,6 \%, 63 / 96)$ say they are currently closed due to the pandemic. Likewise, half of the nursing services $(45,8 \%, 253 / 552)$ reported that care with reduced use of services was at risk/unstable or even not ensured. Conclusions: The results show that the situation of care-dependent people served by home-care services in the present pandemic - and also with a view to a possible second wave - should receive increased attention particularly with regard to unstable/ risky care arrangements.
\end{abstract}

Keywords: home care; COVID-19; long-term care; nursing staff; protective material 
Was ist (zu dieser Thematik) schon bekannt?

Deutschlandweit fehlen Aussagen zur Situation ambulant versorgter Pflegebedürftiger während der COVID-19-Pandemie.

\section{Was ist neu?}

Die Studie liefert erstmals umfangreiche Daten in Bezug auf Erkrankungsfälle, strukturelle Ressourcen und zur Versorgungssituation.

Welche Konsequenzen haben die Ergebnisse für die Pflegepraxis?

Ambulanter Pflege sollte - auch mit Blick auf eine mögliche zweite Pandemie-Welle - eine erhöhte Aufmerksamkeit in Bezug auf prekäre Versorgungssituationen zukommen.

\section{Einleitung}

Die COVID-19-Pandemie geht aktuell weltweit mit gravierenden Auswirkungen für die Gesellschaft und insbesondere das Gesundheitssystem einher. Für Deutschland wurden zum Stichtag 05. Mai diesen Jahres 163820 laborbestätigte COVID-19-Fälle an das Robert Koch-Institut (RKI) übermittelt (RKI, 2020a), darunter 6831 Todesfälle in Zusammenhang mit COVID-19 (RKI, 2020c). Während gesamtgesellschaftlich eine Sterberate von 4,2\% (Stand 05. Mai 2020) ermittelt wurde, waren $87 \%$ dieser Todesfälle und $19 \%$ aller infizierten Personen 70 Jahre oder älter. Das Durchschnittsalter der Verstorbenen liegt bei 81 Jahren (RKI, 2020c) (der Stichtag 05. Mai 2020 wurde ausgewählt, da zu diesem Zeitpunkt die Hälfte aller an dieser Studie teilnehmenden ambulanten Pflegedienste und teilstationären Einrichtungen den Online-Fragebogen ausgefüllt hatten). Da vor allem ältere Menschen bei einer Infektion mit dem SARS-CoV-2-Virus von schweren Krankheitsverläufen und einer hohen Mortalität betroffen sind, soll in der vorliegenden Studie in Ergänzung zum Setting der vollstationären Langzeitpflege (siehe hierzu auch Rothgang et al., 2020) die Situation in der ambulanten und teilstationären Pflege analysiert werden.

Menschen mit Pflegebedarf, die ambulant versorgt werden, haben aufgrund ihres in der Regel höheren Alters und bestehender Pflegebedürftigkeit ein erhöhtes Risiko für einen schweren COVID-19-Krankheitsverlauf (RKI, 2020b). Ihre Anzahl ist mit 829958 Personen (Statistisches Bundesamt, 2018) ähnlich groß wie die Anzahl der in der stationären Langzeitversorgung lebenden Personen. Pflegebedürftige, die ambulant versorgt werden, weisen zwar durchschnittlich einen geringeren Pflegebedarf auf als vollstationär versorgte Personen, dennoch ist die Zahl der Klient_innen mit hohem Pflegebedarf (Pflegegrad 3, 4 oder 5) mit 396245 Personen (47,7\% aller ambulant versorgten Personen) groß (Statistisches Bundesamt, 2018). In den bundesweit 14050 Pflegediensten, die Leistungen nach (deutschem) Sozialgesetzbuch (SGB) V, XI und XII erbringen, sind 390322 Mitarbeitende beschäftigt (Statistisches Bundesamt, 2018), die im Rahmen ihrer Tätigkeit mit direktem Personenkontakt ebenfalls einem besonders hohen Risiko an COVID-19 zu erkranken ausgesetzt sind.

Bereits vor Ausbruch der Pandemie bestanden in der ambulanten Langzeitpflege Herausforderungen durch eine unzureichende Personalausstattung (Büscher, Schröder, Gruber, Bäumker \& Zeiser, 2020). Durch die Pandemie ergeben sich nun, neben der Kompensation von Personalausfällen, zusätzliche Anforderungen durch veränderte Arbeitsbedingungen, z.B. aufgrund notwendiger Hygienerichtlinien und Handlungsempfehlungen, die die Arbeitsbelastung beruflich Pflegender verdichten. Empfehlungen für einen ressourcenschonenden Einsatz von Mund-Nasen-Schutz (MNS) und FFP-Masken in Einrichtungen des Gesundheitswesens bei Lieferengpässen im Zusammenhang mit der neuartigen SARS-CoV-2-Virus-Erkrankung COVID-19 (RKI, 2020c) verstärken zudem bei beruflich Pflegenden die Sorge vor potenziellen Infektionen (Begerow \& Gaidys, 2020).

Menschen mit Pflegebedarf erfahren während der Pandemie Einschränkungen in ihrer gewohnten Versorgung, da beispielsweise teilstationäre Einrichtungen (Tages- und Nachtpflege) nach landesspezifischen Vorgaben vorübergehend geschlossen wurden und eine teilstationäre Pflege nur noch im Sinne einer Notbetreuung in Anspruch genommen werden kann (Ministerium für Arbeit, Gesundheit und Soziales des Landes Nordrhein-Westfalen, 2020). Pflegebedürftige in der ambulanten Versorgung nehmen zudem oftmals zusätzlich Unterstützung von Migrant_innen aus Mittel- und Osteuropa in Anspruch. Die Zahl an migrantischen Betreuungskräften wird auf 700000 Personen in 250000 Haushalten geschätzt, wobei pro Haushalt innerhalb eines Jahres zwei bis drei Hilfskräfte arbeiten (Petermann, Jolly \& Schrader, 2020), von denen viele aufgrund der Pandemie und den damit verbundenen Reisebeschränkungen in ihre Herkunftsländer zurückgekehrt sind (Dibelius \& Piechotta-Henze, 2020).

Ambulant versorgte Pflegebedürftige müssen vor dem geschilderten Hintergrund aktuell massive Herausforderungen bewältigen, und auch Pflegedienstleitungen sind besonders gefordert, bei oftmals knappen personellen und sachlichen Ressourcen sowie neuen Anforderungen in Bezug auf Versorgungsstandards, eine qualitativ hochwertige Versorgung sicherzustellen und ihrer Fürsorgepflicht für Beschäftigte gerecht zu werden. Zusammengefasst ist die Situation in der ambulanten Pflege also durch mehrfache Dilemmata gekennzeichnet: Eine sehr große Anzahl von Personen mit einer zum Teil hohen Vulnerabilität ist von der Pandemie betroffen und einfach durchzuführende Schutzmaßnahmen wie soziale Distanz sind im Bereich der direkten Versorgung nicht anwendbar, sodass ein erhöhtes Infektionsrisiko mit den beschriebenen Folgen besteht. Zusätzlich sind die negativen Folgen einer sozialen Isolierung von Menschen mit Pflegebedarf versus Infektionsschutzmaßnahmen abzuwägen und zu bewerten.

Derzeit fehlen valide Daten zur Situation in ambulanten Pflegediensten und teilstationären Einrichtungen, die es Leistungserbringern aber auch politischen Akteuren ermöglichen, auf die Herausforderungen und Unterstüt- 
zungsbedarfe aufgrund der Pandemie organisatorisch und administrativ zu reagieren. Dies ist umso wichtiger, da derzeit noch kein Impfstoff zur Verfügung steht.

\section{Fragestellungen und Ziele}

Ziel der Studie ist es,

- darzustellen, in welchem Umfang ambulante und teilstationäre Pflegeeinrichtungen zum Erhebungszeitpunkt Anfang Mai 2020 Verdachtsfälle, laborbestätigt infizierte bzw. verstorbene Pflegebedürftige und Mitarbeitende aufweisen,

- die personellen und materiellen Ressourcen der Pflegedienste bzw. deren Mangel zu beschreiben,

- die Versorgungssituation von ambulant versorgten Pflegebedürftigen zu beleuchten,

- den organisatorischen Umgang mit der Situation darzustellen und

- die Unterstützungswünsche der Pflegedienste an die Politik zu skizzieren.

Die Ergebnisse sollen dazu dienen, die besondere Situation von Pflegediensten aber auch Pflegebedürftigen während der Pandemie besser zu verstehen sowie deren zielgenaue Unterstützung durch politische Entscheidungsträger zu ermöglichen. Gleichzeitig tragen die Ergebnisse dazu bei, die Vorbereitung auf eine mögliche zweite Welle der Pandemie zu unterstützen.

\section{Methode und Material}

\section{Studiendesign}

Die vorliegende Querschnittstudie wurde durch die Universität Bremen vom 28. April bis zum 12. Mai 2020 deutschlandweit in ambulanten Pflegediensten und teilstationären Einrichtungen durchgeführt. Eingesetzt wurde ein im Rahmen dieser Studie entwickelter OnlineFragebogen. Die vorliegend berichtete Studie ist Teil einer gemeinsam konzipierten Studie, die auch den Bereich der stationären Einrichtungen umfasst, über die gesondert an anderer Stelle berichtet wird (Rothgang et al., 2020). Die Items der Onlinebefragung generieren sich aus projektinternen Literaturrecherchen und Vorarbeiten des Studienteams, die Beantwortungszeit betrug ca. 20 Minuten. Der Fragebogen enthält sowohl geschlossene Fragen (Einfachoder Mehrfachauswahl) als auch offene Fragen (Freitextformat) und umfasst neben Strukturmerkmalen der Pflegedienste/Einrichtungen vier Fragenkomplexe zum Vorkommen des SARS-CoV-2-Virus in den Pflegediensten/Einrichtungen, Auswirkungen der Pandemie, z.B. in Bezug auf personelle und sachliche Ausstattung aber auch $\mathrm{zu}$ veränderten Arbeitsprozessen und Kommunikationsstrukturen. Zudem wurde den Befragten die Gelegenheit gegeben, ihre Wünsche an die Politik zu äußern.
Ein Link zur Onlinebefragung wurde per E-Mail an eine Gelegenheitsstichprobe von 9547 ambulanten Pflegediensten und 2731 teilstationären Einrichtungen versandt. Darüber hinaus wurde die Befragung über Kontakte des Studienteams zu Interessensvertretungen und Anbieterverbänden beworben. Eine Erinnerung zur Teilnahme erfolgte eine Woche nach der ersten Einladung per E-Mail. Die Onlinebefragung erfolgte mittels EFS-Survey, Version Fall 2019 (Questback GmbH, 2019). Den potenziell Teilnehmenden wurde vorab ein Informationsschreiben mit einer Erläuterung der Studie und des Datenschutzes zur Verfügung gestellt. Ein Datenschutzkonzept wurde mit der Datenschutzbeauftragten der Universität Bremen abgestimmt. Alle Teilnehmenden mussten der Datenschutzerklärung zustimmen.

\section{Datenaufbereitung und -auswertung}

Zunächst gingen alle Datensätze von Personen in die Analyse ein, welche die Befragung begonnen hatten (755 Pflegedienste, 101 teilstationäre Einrichtungen). In einem ersten Schritt wurden Teilnehmende ausgeschlossen, die ausschließlich Angaben zu ihrem Pflegedienst/ihrer Einrichtung, nicht jedoch zu themenbezogenen Fragen machten (54 Pflegedienste, 5 teilstationäre Einrichtungen). Die Befragungsdaten der verbliebenen 701 Pflegedienste und 96 teilstationären Einrichtungen gingen in die Auswertung ein und wurden vor der Auswertung je Item auf Plausibilität geprüft. Antworten außerhalb des gültigen Wertebereichs wurden dann ebenso wie fehlende Angaben jeweils für dieses Item aus der Auswertung ausgeschlossen. Das für die Prozentberechnung verwendete $\mathrm{N}$ der berücksichtigten, gültigen Fälle wird deshalb bei der Ergebnisdarstellung individuell angegeben. Angaben von absoluten Häufigkeiten werden mit n gekennzeichnet. Die Auswertung erfolgte deskriptiv unter Verwendung von Häufigkeiten, Mittelwerten und Chi-Quadrat-Unabhängigkeitstests (nominales Signifikanzniveau $\alpha=0,05$ ). Die in Freitexten gegebenen Antworten wurden zusammenfassend inhaltsanalytisch gebündelt und in ein Kategoriensystem überführt. Die statistische Auswertung wurde mit der Software SAS Version 9.4 durchgeführt.

\section{Ergebnisse}

\section{Beschreibung der Stichprobe}

Für die Auswertung finden Datensätze von insgesamt 701 Pflegediensten und 96 teilstationären Einrichtungen Verwendung. Bezogen auf die initial angeschriebenen 9547 ambulanten Pflegedienste und 2731 teilstationären Einrichtungen entspricht dies einem Rücklauf von rund 7,3\% bzw. 3,5\%. Dabei antwortete in einem Drittel der Fälle die Einrichtungsleitung und in zwei Dritteln der Fälle die Pflegedienstleitung. Zum Zeitpunkt der Befragung waren in 
den Pflegediensten 26261 (teilstationäre Einrichtungen: 2599) Mitarbeitende beschäftigt, die (in)direkt für die Versorgung von 82949 Klient_innen (2236 Gäste) zuständig waren. Die durchschnittliche Anzahl von Klient_innen liegt damit bei 118,3 Personen, ist aber aufgrund der fehlenden Differenzierung nach SGB V/XI/XII-Leistungen nur eingeschränkt mit Werten der amtlichen Pflegestatistik vergleichbar. 42,7\% (291/682) der Pflegedienste erbringen Leistungen im betreuten Wohnen und 23,9\% (162/678) in ambulant betreuen Wohngemeinschaften. Die Pflegedienste in der Stichprobe sind gemessen an der Zahl der Klient_innen und der Zahl an Mitarbeitenden (siehe Tab. 1) deutlich größer als Pflegedienste im Bundesdurchschnitt. Öffentliche Träger sind in der Stichprobe im
Gegensatz zur Grundgesamtheit etwas über- und private Träger etwas unterrepräsentiert. Alle Bundesländer sind weitgehend entsprechend ihrer Verteilung in der Pflegestatistik vertreten.

Die durchschnittliche Anzahl von Plätzen und Gästen in den teilstationären Einrichtungen liegt bei 19,0 bzw. 23,3. Die Einrichtungen in der Stichprobe sind damit etwas größer als Einrichtungen im Bundesdurchschnitt (siehe Tab.1). Fast jede sechste Einrichtung (15,6\%, $15 / 96)$ ist dabei schwerpunktmäßig auf die Versorgung von Menschen mit Demenz spezialisiert. Teilstationäre Einrichtungen sind aus fast allen Bundesländern vertreten (mit Ausnahme von Bremen, Rheinland-Pfalz und dem Saarland). Sie befinden sich in jeweils knapp der

Tabelle 1. Vergleich der Studienergebnisse mit der amtlichen Statistik für ambulante Pflegedienste und teilstationäre Einrichtungen

\begin{tabular}{|c|c|c|c|}
\hline Merkmal & \multicolumn{2}{|c|}{ Studienergebnis } & $\begin{array}{l}\text { Amtliche Statistik } \\
\text { (Statistisches Bundesamt, 2018) }\end{array}$ \\
\hline \multicolumn{4}{|l|}{ Ambulante Pflegedienste } \\
\hline \multicolumn{4}{|l|}{ Mitarbeitende (Mittelwert) } \\
\hline Personenzahl (N = 613) & \multicolumn{2}{|c|}{37,5} & 27,8 \\
\hline Vollzeitäquivalente ( $N=579)$ & \multicolumn{2}{|c|}{19,8} & 18,9 \\
\hline \multicolumn{4}{|l|}{ Pflegekräfte (Mittelwert) } \\
\hline Personenzahl (N = 595) & \multicolumn{2}{|c|}{25,8} & 20,1 \\
\hline Vollzeitäquivalente (N = 543) & \multicolumn{2}{|c|}{15,5} & 13,9 \\
\hline \multicolumn{4}{|l|}{ Trägerschaft ( $N=674)$} \\
\hline Öffentlich & \multicolumn{2}{|c|}{$5,0 \%(34)$} & $1,4 \%$ \\
\hline Freigemeinnützig & \multicolumn{2}{|c|}{$33,8 \%(228)$} & $32,8 \%$ \\
\hline Privat & \multicolumn{2}{|c|}{$61,1 \%(412)$} & $65,8 \%$ \\
\hline Gesamtzahl ... & $\begin{array}{l}\text { Stichprobe } \\
(\mathrm{N}=701)\end{array}$ & Hochrechnung1 & RKI \\
\hline infizierter Klient_innen & 250 & 2.499 & - \\
\hline verstorbener Klient_innen & 81 & 784 & - \\
\hline genesener Klient_innen & 113 & 1.127 & - \\
\hline infizierter Mitarbeiter_innen & 116 & 1.717 & - \\
\hline genesener Mitarbeiter_innen & 98 & 1.462 & - \\
\hline \multicolumn{4}{|l|}{ Teilstationäre Einrichtungen } \\
\hline \multicolumn{4}{|l|}{ Einrichtungsgröße (Mittelwert) } \\
\hline Zahl der Plätze (N = 94) & & & 14,4 \\
\hline Gäste (N = 92) & & & 22,5 \\
\hline \multicolumn{4}{|l|}{ Mitarbeitende (Mittelwert) } \\
\hline Personenzahl ( $N=95)$ & & & - \\
\hline Vollzeitäquivalente ( $\mathrm{N}=83$ ) & & & - \\
\hline \multicolumn{4}{|l|}{ Pflegekräfte (Mittelwert) } \\
\hline Personenzahl (N = 91) & & & - \\
\hline Vollzeitäquivalente ( $N=84)$ & & & - \\
\hline \multicolumn{4}{|l|}{ Trägerschaft $(\mathrm{N}=92)$} \\
\hline öffentlich & \multicolumn{2}{|c|}{$7,3 \%(7)$} & - \\
\hline freigemeinnützig & \multicolumn{2}{|c|}{$45,8 \%(44)$} & - \\
\hline privat & \multicolumn{2}{|c|}{$42,7 \%(41)$} & - \\
\hline
\end{tabular}

Anmerkungen: ${ }^{1}$ Die Werte aus der vorliegenden Stichprobe wurden anteilsmäßig auf die Anzahl aller Pflegebedürftigen in Deutschland hochgerechnet: Hierzu wurde jeweils für Pflegebedürftige und Mitarbeitende ein Hochrechnungsfaktor errechnet aus bundesweiter Anzahl nach Pflegestatistik 2017 / Anzahl in der Stichprobe und die Fälle aus der Stichprobe mit diesem Faktor multipliziert. 
Hälfte aller Fälle in freigemeinnütziger $(47,8 \%, 44 / 92)$ oder privater $(44,6 \%, 41 / 92)$ Trägerschaft $(7,6 \%$ öffentliche Trägerschaft, 7/92). Daten zur Trägerschaft für teilstationäre Eirichtungen sind in der amtlichen Statistik nicht vorhanden - vergleicht man die Angaben mit denen stationärer Einrichtungen, so sind öffentliche Träger in der Stichprobe etwas über- und freigemeinnützige Träger etwas unterrepräsentiert. Sechs von zehn Einrichtungen geben an, aufgrund der vorliegenden Pandemie derzeit geschlossen zu sein $(60,0 \%, 57 / 95)$.

\section{Verbreitung von COVID-19 in ambulanten Pflegediensten}

Tabelle 2 enthält die Zahl der ambulanten Pflegedienste $(\mathrm{N}=627)$, bei denen Angaben zu bestätigten Fällen von COVID-19 und / oder Verdachtsfälle für Klient_innen und Mitarbeitende vorhanden sind. Demnach haben 16,7\% (105/627) der Pflegedienste Klient_innen mit einer bestätigten Infektion (und ggf. zusätzlich Verdachtsfälle) und weitere 13,4\% (84/627) der Dienste haben mindestens einen Verdachtsfall unter den Klient_innen. Insgesamt sind damit drei von zehn Diensten $(30,1 \%, 189 / 627)$ von Klient_innen mit COVID-19-Verdacht oder -Infektion betroffen. Mehr als jeder 12. aller antwortenden Dienste $(8,7 \%, 52 / 599)$ hat zumindest einen COVID-19-Todesfall unter seinen Klient_innen. Bei den Mitarbeitenden liegen die Werte an labormedizinisch bestätigt infizierten Mitarbeitenden dagegen deutlich geringer $(8,9 \%, 56 / 627)$. Mit jedem fünften Dienst $(20,4 \%, 128 / 627)$, der auf Seiten von Klient_innen oder Mitarbeitenden und jedem 20. Dienst (5,3\%, 33/627), der sowohl bei Klient_innen und Mitarbeitenden bestätigte COVID-19-Fälle aufweist, sind ambulante Pflegedienste damit deutlich von der Pandemie betroffen. Die Situation bei Mitarbeitenden und Klient_innen ist dabei nicht unabhängig voneinander (Chi-Quadrat-Unabhängigkeitstest, $\mathrm{p}<0,001$ ). Für etwa sechs von zehn Diensten mit Mitarbeiterfällen (58,9\%; 33 von 56 Diensten) gilt, dass auch bei Klient_innen bestätigte Fälle (durchschnittlich 1,3 Fälle) bekannt sind, umgekehrt gilt bei bestätigten Fällen von Klient_innen, dass nur bei drei von zehn Diensten (31,4\%, 33 von 105 Diensten) auch Fälle unter den Mitarbeitenden bekannt sind - Mitarbeitende haben also bei Erkrankungsfällen unter Klient_ innen ein erhöhtes eigenes Erkrankungsrisiko.
Durchschnittlich berichten von COVID-19 betroffene Dienste dabei von jeweils zwei infizierten Klient_innen/Mitarbeitenden $(n=84$ und $n=42)$ und jeweils drei weiteren Verdachtsfällen (jeweils $n=93$ ). Knapp jede / jeder dritte positiv auf SARS-CoV-2 getestete Klient_in $(32,4 \%, 81 / 250)$ ist verstorben. Zwei Drittel $(65,8 \%)$ der positiv getesteten Klient_innen (Daten aus 71 Diensten mit bestätigten Fällen bei Klient_innen) und 84,6\% der Mitarbeitenden (Daten aus 39 Diensten mit bestätigten Fällen bei Mitarbeitenden) weisen im Mittel typische Symptome der Erkrankung auf. Sowohl bei Klient_innen als auch bei Mitarbeitenden liegt die durchschnittliche Zeit von einer Testung bis zur Bekanntgabe des Ergebnisses bei etwa vier Tagen (Klient_innen 4,5 Tage, $\mathrm{n}=63$; Mitarbeitende 3,9 Tage, $\mathrm{n}=133)$.

In teilstationären Einrichtungen berichten lediglich vier der 96 Einrichtungen von bestätigten COVID-19-Fällen $(4,2 \%)$, die Gesamtzahl aller bestätigten Fälle beläuft sich auf $\mathrm{n}=13$ Tagesgäste, die der Verstorbenen auf sechs. Drei Einrichtungen berichten zudem über Infektionen unter den Mitarbeitenden, dann jedoch gleich über mehr als einen Fall. Aufgrund der geringen Einrichtungs- und Fallzahlen wird auf eine detailliertere Auswertung verzichtet.

\section{Personelle und materielle Ressourcen}

Vor Ausbruch der Pandemie lag der Umsatzanteil an SGB VLeistungen bei den ambulanten Pflegediensten durchschnittlich bei etwas weniger als der Hälfte $(45,3 \%, n=564$ antwortende Dienste) aller Umsätze, der der SGB XI-Leistungen knapp darüber $(50,9 \%, \mathrm{n}=571$ antwortende Dienste). Im Zuge der Pandemie ändert sich die Inanspruchnahme von SGB V-Leistungen bei etwa der Hälfte $(47,6 \%$, 288/605) der Pflegedienste, hiervon sind drei Viertel $(73,6 \%, 212 / 288)$ von einer Abnahme betroffen. Ein ähnliches Bild zeigt sich bei der Inanspruchnahme von Grundpflegeleistungen nach SGB XI. Mehr als die Hälfte aller antwortenden Pflegedienste $(59,7 \%, 375 / 628)$ berichten von Veränderungen, wobei in vier von fünf Fällen $(77,9 \%$, 292/375) eine Abnahme an Leistungen stattfindet. Eine deutliche Abnahme von Leistungen wird auch für die Inanspruchnahme von Betreuungsmaßnahmen $(65,4 \%$, $395 / 604)$, hauswirtschaftlicher Versorgung (64,2\%, $385 / 600)$, Service- $(44,1 \%, 235 / 533)$ und Beratungsleistungen $(65,5 \%, 392 / 617)$ berichtet. Mehr als die Hälfte der

Tabelle 2. Von COVID-19 betroffene Klient_innen und Mitarbeitende in ambulanten Pflegediensten $(n=627)$

\begin{tabular}{|c|c|c|c|c|c|}
\hline & & \multicolumn{4}{|c|}{ Mitarbeitende } \\
\hline & & infiziert & Verdacht & kein Fall & insgesamt \\
\hline \multirow[t]{4}{*}{ Klient_innen } & infiziert & $5,3 \%$ & $4,6 \%$ & $6,9 \%$ & $16,7 \%$ \\
\hline & Verdacht & $1,0 \%$ & $4,0 \%$ & $8,5 \%$ & $13,4 \%$ \\
\hline & kein Fall & $2,7 \%$ & $9,9 \%$ & $57,3 \%$ & $69,9 \%$ \\
\hline & insgesamt & $8,9 \%$ & $18,5 \%$ & $72,6 \%$ & $100,0 \%$ \\
\hline
\end{tabular}

Anmerkungen: Infiziert = mindestens ein bestätigter Fall, Verdacht = mindestens ein Verdachtsfall, aber kein bestätigter Fall, Kein Fall = weder ein bestätigter, noch ein Verdachtsfall. 
Pflegedienste (59,9\%, 354/591) erwarten aufgrund der Pandemiefolgen Umsatzverluste. Von einem Personalausfall von bis zu zehn Prozent (einschließlich etwaiger Quarantänemaßnahmen) berichten etwas weniger als die Hälfte der Pflegedienste (43,0\%, 262/609) für den Monat April 2020 , ebenso viele waren nicht betroffen von einem solchen Ausfall (42,7\%, 260 / 609). Durchschnittlich wird aufgrund der Pandemie und damit veränderter Arbeitsbedingungen von etwa zwei Dritteln der Dienste (63,8\%, 365/572) ein Arbeitsmehraufwand berichtet, der mit durchschnittlich 40 Minuten pro Schicht beziffert wird $(\mathrm{n}=347)$.

Von einem Personalausfall berichten mehr als ein Viertel aller teilstationären Einrichtungen $(28,1 \%$; $n=96)$, jede zehnte Einrichtung ( $n=96)$ sogar von Personalausfällen von mehr als 20 Prozent (einschließlich etwaiger Quarantänemaßnahmen) für den Monat April 2020. Zwei Drittel der Einrichtungen (65,6\%, 63/96) berichten von einem Aufnahmestopp bzw. sind ganz geschlossen.

Von einem Mangel an persönlicher Schutzausrüstung (bis zu einmal pro Woche oder seltener) im Verlauf der bisherigen Pandemie-Situation berichten über die Hälfte der Dienste $(55,6 \% 324 / 583)$ bzw. fast die Hälfte der teilstationären Einrichtungen (42,6\% 26/61), etwas mehr als jeder dritte Dienst $(34,3 \%, 194 / 566)$ bzw. jede fünfte Einrichtung $(34,4 \%, 22 / 64)$ berichtet dies auch für Flächendesinfektionsmittel. Ein Vergleich über die Zeit (für ambulante Pflegedienste siehe Abb. 1) zeigt jedoch auch, dass sich diese Mangelsituation in der letzten Woche vor der Befragung bereits wieder etwas entspannt (Schutzausrüstung: 24,0\%, 134/574, Flächendesinfektionsmittel: 10,9\%, 61/558). Für teilstationäre Einrichtungen erfolgt kein Vergleich aufgrund der teilweisen Einrichtungsschließungen.

\section{Auswirkungen auf Pflegebedürftige}

Nach Auskunft aus den ambulanten Pflegediensten lebten vor Ausbruch der Pandemie bei 41,8\% ( $n=293)$ aller 701 Dienste in mindestens einem von ihnen versorgten Haushalt bezahlte Hilfs-/ Betreuungskräfte aus dem Ausland, bei etwa ebenso vielen war dies nicht der Fall $(42,2 \%, n=296)$ und für $16,0 \%(n=112)$ aller Pflegedienste lagen hierzu keine Informationen vor. Durchschnittlich wurden 6,3 Haushalte mit bezahlten Hilfs-/Betreuungskräften aus dem Ausland genannt, was einer Gesamtzahl von 1837 Haushalten in der vorliegenden Stichprobe entspricht. Nach Ausbruch der Pandemie berichten ein Drittel der Pflegedienste (32,4\%, $95 / 293)$, dass in den von ihnen versorgten Haushalten diese Hilfs- und Betreuungskräfte nun fehlen, dies betrifft anteilmäßig nach Schätzungen aus den Pflegediensten die Hälfte aller von ihnen versorgten Haushalte mit einer zuvor vorhandenen Hilfs- und Betreuungskraft. Aufgrund der zuvor schon berichteten Rückgänge in den in Anspruch genommenen Leistungen sowie derzeit fehlender teilstationärer Versorgungsangebote (s.o.) schätzt fast die Hälfte aller Pflegedienste $(45,8 \%, 253 / 552)$ die Versorgung von Personen mit geringer Inanspruchnahme von Leistungen als gefährdet/instabil oder sogar nicht sichergestellt ein.

\section{Umgang mit dem Virus}

Zwei Drittel aller befragten Pflegedienste (65,3\%, 458/701) (und mehr als die Hälfte aller teilstationären Einrichtungen: 58,3\%, 56/96) geben an, dass sie vor Ausbruch der COVID-19-Pandemie nicht speziell auf die Situation einer Pandemie vorbereitet gewesen sind - auch im Rahmen von Ausbildung/Studium (9,3\%, 65/701 und 2,3\%, 16/701) oder aber auch Fortbildungen (13,3\%, $93 / 701)$ ist dieses Thema bisher in den Pflegediensten eher selten präsent. Zur Bewältigung der vorliegenden Pandemie-Situation haben mehr als zwei Drittel $(68,1 \%$, 477 / 701) aller Pflegedienste (und mehr als die Hälfte aller teilstationären Einrichtungen: 56,3\%, 54/96) ein Krisenteam gegründet, jeweils etwa ein Sechstel aller Dienste hat

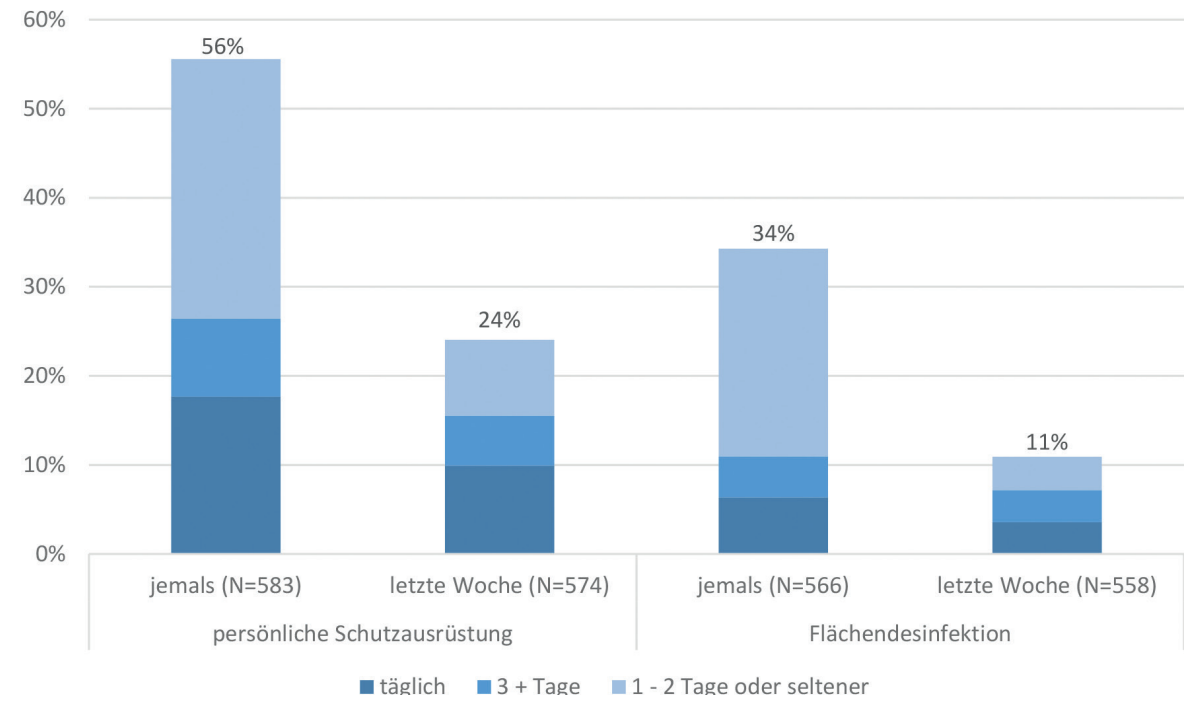

Abbildung 1. Mangel an Schutzmaterialien in ambulanten Pflegediensten. 


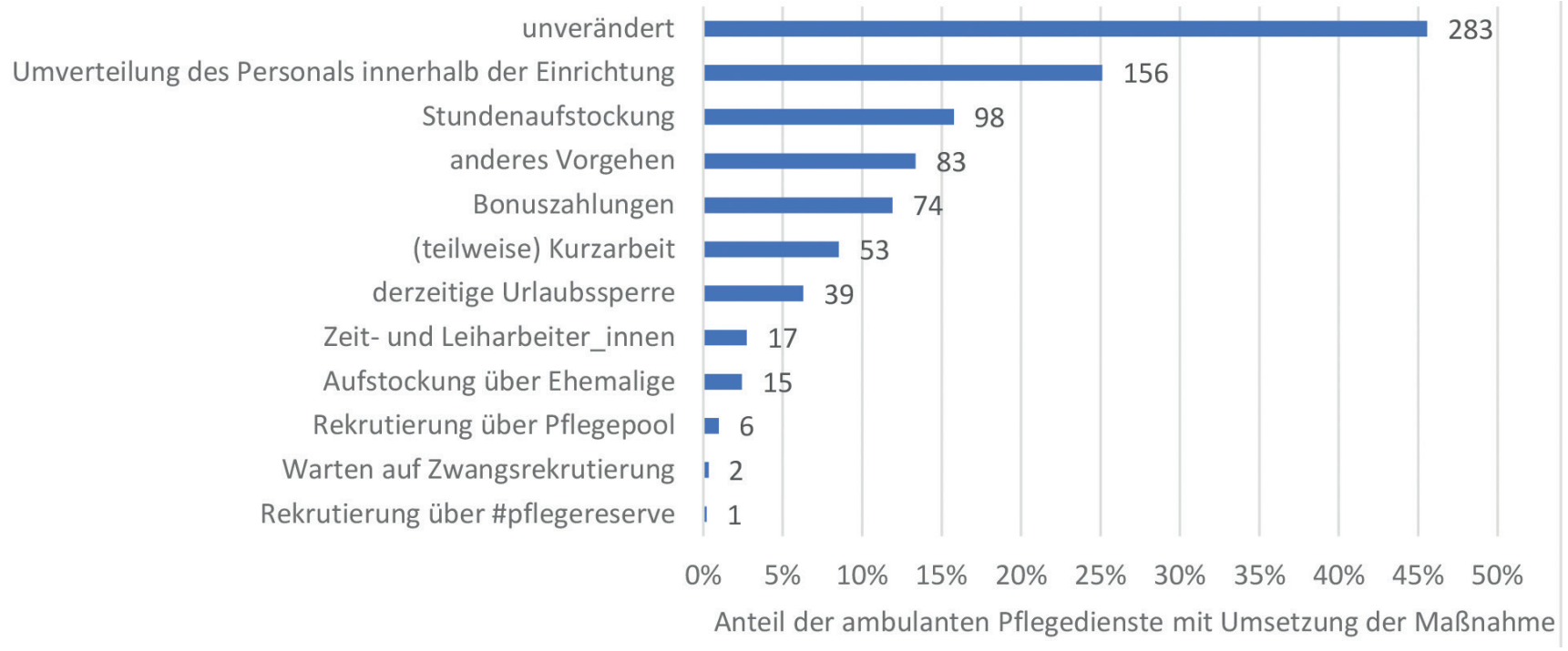

Abbildung 2. Maßnahmen zum Personalmanagement in ambulanten Pflegediensten (Mehrfachnennungen möglich).

dies nicht getan $(16,1 \%, 113 / 701)$ oder keine Angabe hierzu gemacht $(15,8 \%, 111 / 701)$.

Knapp $90 \%$ aller Dienste (621/701) machten Angaben über Maßnahmen zum Personalmanagement während der Pandemie (siehe Abb. 2). Etwas weniger als die Hälfte dieser Dienste $(45,6 \%, 283 / 621)$ haben kein verändertes Personalmanagement bei ausreichendem Personal, gefolgt von einem Viertel der Dienste (25,1\%, 156/621), die Versorgungssituationen über die Umverteilung des Stammpersonals sicherstellen. Eine Aufstockung über Ehemalige, die Rekrutierung über einen Pflegepool oder \#pflegereserve spielt nur eine (sehr) geringe Rolle. Auffallend ist, dass es sowohl Dienste gibt, die einen gestiegenen Arbeitsaufwand bewältigen müssen (z.B. über Stundenaufstockungen, Urlaubssperren etc.), andererseits aber fast jeder elfte Dienst $(8,5 \%, 53 / 621)$ auch über (teilweise) Kurzarbeit berichtet.

Bei den teilstationären Einrichtungen berichtet mehr als ein Drittel der Einrichtungen (18,4\%, 16/87), dass sie kein verändertes Personalmanagement bei ausreichendem Personal haben. Auffallend ist, dass fast jede dritte Einrichtung $(32,2 \%, 28 / 87)$ über (teilweise) Kurzarbeit berichtet.

Knapp drei Viertel aller Dienste $(72,6 \%, 509 / 701)$ reagieren auf die veränderte Versorgungssituation mit speziellen (geplanten) Schulungen zu den Thema Schutzkleidung und Hygiene, in der Regel sind dies Schulungsangebote mit einer Dauer von bis zu zwei Stunden. Abwesenheitsregeln für Beschäftigte bei COVID-19 typischen Symptomen berichten drei Viertel $(75,5 \%, 529 / 701)$ aller Dienste, einer von 17 Diensten $(5,7 \%, 40 / 701)$ hat solche Regelungen jedoch explizit nicht. Für Beschäftigte, die einer Risikogruppe zugeordnet werden können, hat nur gut ein Viertel $(28,8 \%$, 202/701) aller Dienste Abwesenheitsregeln getroffen. Klient_innen und deren Angehörige werden in mehr als drei Vierteln $(77,3 \%, 542 / 701)$ aller Dienste zu erforderlichen Maßnahmen im Umgang mit COVID-19 geschult. Ein klinisches Monitoring entsprechend der Empfehlung des RKIs
(z.B. tägliche Erfassung und Dokumentation der entsprechenden klinischen Symptome einschließlich der Messung der Körpertemperatur) von Klient_innen auf COVID-19 berichtet ein knappes Drittel (29,8\%, 209/701 oder 33,8\%, $237 / 701$ inkl. Monitoring in Planung) aller Dienste. Etwa ein Drittel $(32,2 \%, 226 / 701)$ aller befragten ambulanten Pflegedienste berichten aufgrund von veränderten Arbeitsund Personalbedingungen auch von einem aktuellen Aufnahmestopp für neue Klient_innen.

\section{Forderungen an die Politik}

Auf die Frage, wie sie die Unterstützung durch übergeordnete Institutionen während der Pandemie beurteilen, zeichnen die Dienste ein überwiegend positives Bild (siehe Abb. 3). Hohe Zustimmungsraten (stimme voll oder eher $\mathrm{zu})$ erhalten Trägerverbände $(85,8 \%, 374 / 436)$, Berufsverbände $(73,6 \%, 370 / 503)$ aber auch das RKI $(66,4 \%$, 373/562). Kranken- und Pflegekassen werden mit einer Zustimmungsrate von 40,6\% (227/560) deutlich kritischer beurteilt. In den teilstationären Einrichtungen zeigt sich ein ähnliches Bild.

Pflegedienste und -einrichtungen waren auch bereits vor der Pandemie gefordert, vielfältige Herausforderungen bei der Sicherstellung einer qualitativ hochwertigen Versorgung zu bewältigen. In Bezug auf Wünsche an Kostenträger, die aus Sicht der befragten Dienste dabei unterstützen sollen, die Herausforderungen der aktuellen Pandemie-Situation zu bewältigen, zeigen sich hohe $\mathrm{Zu}$ stimmungsraten (stimme voll oder eher zu) für finanzielle Anreize (für ambulanten Pflegedienste siehe Abb. 4). So stimmen vier von fünf Diensten $(80,8 \%, 453 / 561)$ einer "Corona-Prämie“ für Pflegekräfte und mehr als drei von fünf Diensten $(64,0 \%, 354 / 553)$ einer Prämie für Berufsrückkehrer zu. Auch personelle Unterstützung durch Berufsaussteiger_innen $(59,9 \%, 327 / 546)$ oder ausländische Kräfte $(52,6 \%, 285 / 542)$ treffen auf eine Zustimmung von 
Ich fühle mich während der Pandemie hinreichend unterstützt durch ...

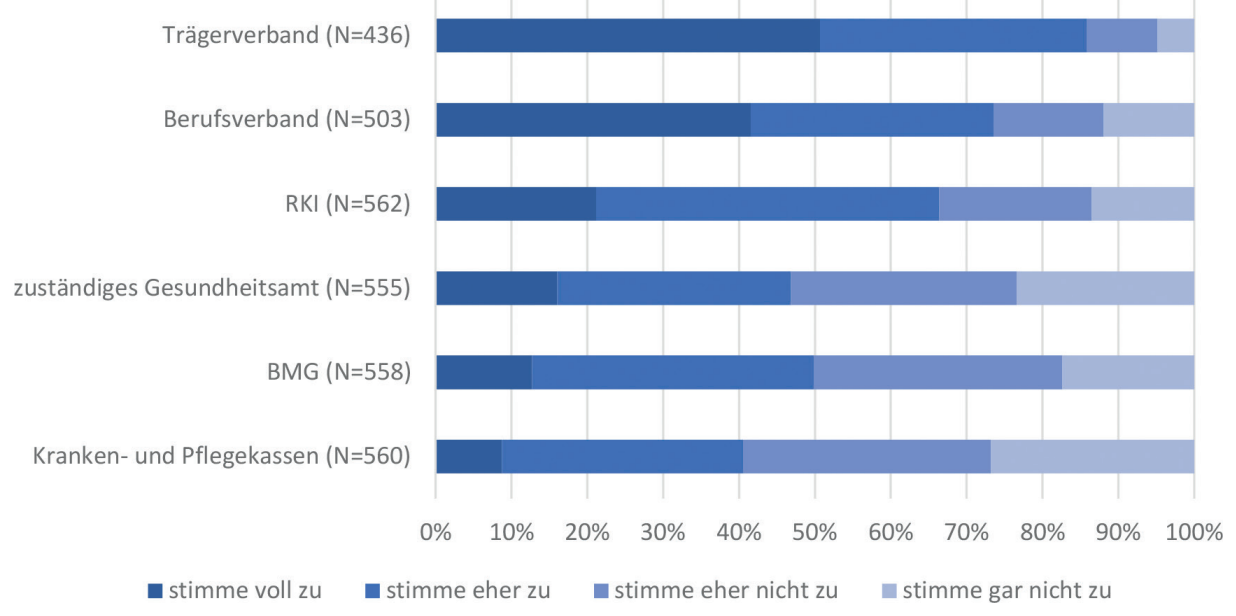

Abbildung 3. Unterstützung ambulanter Pflegedienste durch übergeordnete Institutionen. RKI = Robert Koch-Institut, BMG = Bundesministerium für Gesundheit.

\section{Personalbezogene Unterstützung und Unterstützung im direkten Zusammenhang mit dem Krisenmanagement}

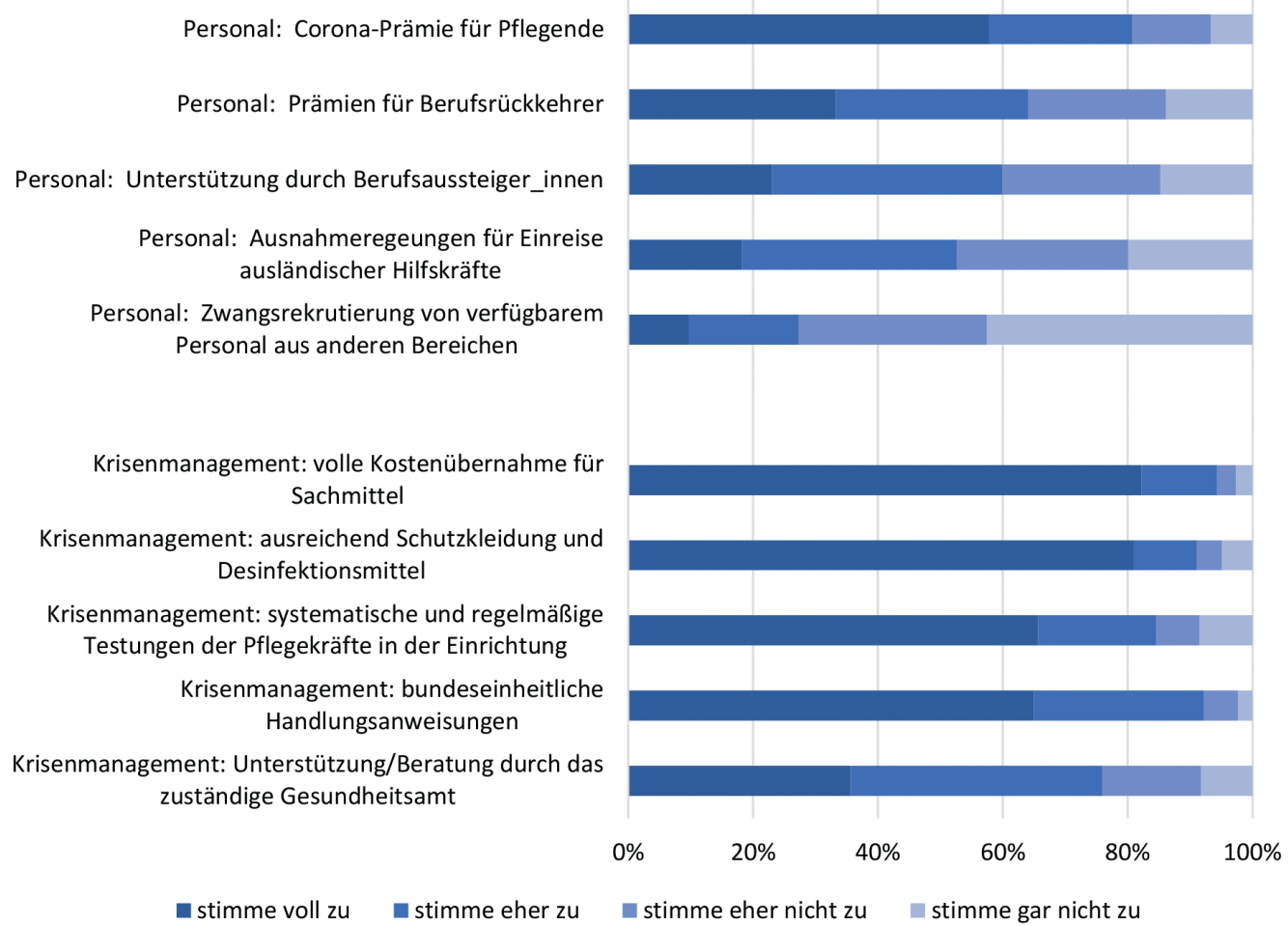

Abbildung 4. Wünsche ambulanter Pflegedienste in Bezug auf eine personalbezogene Unterstützung und Unterstützung im direkten Zusammenhang mit dem Krisenmanagement.

mehr als der Hälfte der Dienste, Zwangsrekrutierungen werden dagegen deutlich abgelehnt.

In Bezug auf das direkte Krisenmanagement (siehe Abb. 4) wird insbesondere die volle Kostenübernahme für Sachmittel (94,3\%), ausreichende Zurverfügungstellung von Schutzmaterialien $(91,0 \%, 530 / 562)$ aber auch bundeseinheitliche Handlungsanweisungen (92,1\%, 515/559) von fast allen Diensten angemahnt. Eine systematische und regelmäßige Testung von Pflegekräften wünschen sich vier von fünf Diensten $(84,5 \%, 465 / 550)$ und drei von vier Diensten $(75,9 \%, 403 / 531)$ eine zugehende dienstbezogene Unterstützung und Beratung bei der Infektionsprävention und -kontrolle in den Diensten durch das zuständige Gesundheitsamt.

Auch in Bezug auf gewünschte Unterstützungsmaßnahmen zeigen sich in den teilstationären Einrichtungen ähnliche Antwortmuster und Zustimmungsraten.

Ein Aussetzen externer Prüfungen während der Pandemie befürworten neun von zehn $(92,4 \%, 520 / 563)$ der Pflegedienste. Darüber hinaus wünschen sich vier von 
fünf Diensten $(85,9 \%, 488 / 568)$ eine Reduktion des Dokumentationsaufwands sowie sieben von zehn Diensten (72,2\%, 389/539) verstärkte Home-Office-Lösungen für administrativ tätige Pflegekräfte.

In einer abschließenden Freitextangabe führten einige Befragten ergänzend aus, dass höhere Mitarbeiterausfälle durch die Arbeitsunfähigkeit der Beschäftigten und ein gestiegener Zeitbedarf in den Einsätzen durch den Mehraufwand zur Durchführung der notwendigen Schutzmaßnahmen entstehen. Zudem sorgen sich einige Dienste, dass gerade der ambulanten Versorgung zu wenig öffentliche Aufmerksamkeit zuteilwird.

\section{Diskussion}

Die vorliegende Studie liefert erstmalig während der aktuellen COVID-19-Pandemie einen Überblick zur Situation von ambulanten Pflegediensten und teilstationären Einrichtungen in Deutschland. Die Stichprobe der Studie basiert dabei auf einer Gelegenheitsstichprobe von 856 ambulanten Pflegediensten und teilstationären Pflegeeinrichtungen und ist nicht zwingend repräsentativ (siehe Limitationen).

Die Studie hat dabei gezeigt, dass ambulante Pflegedienste - aber auch teilstationäre Pflegeeinrichtungen massiv von Auswirkungen der COVID-19-Pandemie zum Erhebungszeitpunkt Anfang Mai 2020 betroffen waren. Während für vollstationäre Pflegeeinrichtungen international hohe Fallzahlen und Sterberaten, etwa aus Spanien (in der Region um Madrid starben im März diesen Jahres allein 4260 Bewohner_innen von Pflegeheimen mit COVID-19 Diagnose oder assoziierten Symptomen), Frankreich und den USA (einer von fünf aller auf COVID-19 zurückzuführenden Todesfällen trat in Pflegeheimen auf) berichtet werden (UN, 2020), nehmen ambulante Pflegedienste in der (inter)nationalen Berichterstattung bislang keine prominente Rolle ein. Die in dieser Studie berichteten Fallzahlen geben einen ersten Einblick in die Verbreitung des SARS-CoV-2-Virus bei ambulant versorgten Pflegebedürftigen. Im Unterschied zur parallel ermittelten Anzahl von infizierten Personen im größenmäßig vergleichbaren stationären Langzeitpflegesetting (siehe hierzu auch Rothgang et al., 2020), sind bundesweit ambulant nur knapp ein Viertel so viele Todessfälle (784 vs. 3345) zu verzeichnen, was vermutlich auf eine höhere Vulnerabilität aber auch größere räumliche Nähe des versorgten Klientels in Pflegeheimen zurückzuführen ist. Eine Einordnung der bestätigten Fallzahlen erweist sich insgesamt als schwierig, da dies zum einen von der Anzahl der durchgeführten Tests abhängig ist und sich zum anderen Hinweise aus Studien mehren, dass vermutlich viele Fälle aufgrund eines Fehlens typischer Symptome nicht als Fälle erkannt bzw. getestet werden (Ing, Cocks \& Green, 2020; Stobbe, 2020). Berücksichtigt man, dass nach derzeitigen Erkenntnissen zudem „eine relevante Infektiosität bereits zwei Tage vor Symptombeginn vorhanden ist und die höchste Infektiosität am Tag vor dem Symptombeginn liegt" (RKI, 2020b), so ergeben sich daraus große Konsequenzen für eine rechtzeitige Testung von Mitarbeitenden in Pflegediensten und -einrichtungen - aber auch für Pflegebedürftige. Die ermittelte derzeitige durchschnittliche Dauer bis zum Bekanntwerden eines Testergebnisses von vier Tagen ist damit als völlig unzureichend zu bewerten.

\section{Personelle und materielle Ressourcen}

Von einem Personalausfall bis zu $10 \%$ durch z. B. Quarantänemaßnahmen berichten rund $45 \%$ der Pflegedienste. Pflegedienste versuchen den personellen Mehraufwand in der Versorgung dabei mehrheitlich durch interne Lösungen aufzufangen. Angesichts der ohnehin sehr angespannten Personalsituation im Bereich der ambulanten Pflege ist dies, neben Umsatzeinbußen, ein Faktor der existenziell bedrohlich sein kann.

Auch für teilstationäre Einrichtungen dürften die berichteten Personalausfälle und pandemiebedingten Mehraufwände bis hin zu Schließungen aufgrund der Pandemie und der daraus resultierenden Mehrkosten und Umsatzausfälle erhebliche bis existenzielle Konsequenzen für die entsprechenden Einrichtungen nach sich ziehen. Eine sich bereits im Rahmen der Umfrage direkt abzeichnende Konsequenz für Mitarbeitende besteht in der großen Anzahl von Einrichtungen (32,2\%), die über Kurzarbeit berichten.

Über die Hälfte der Pflegedienste berichten in der Pandemie von Arbeitsmehraufwand. Schon vor Beginn der Pandemie waren Pflegekräfte häufig psychischen und emotionalen Belastungen sowie schweren körperlichen Anforderungen ausgesetzt - die Pflege zählt zu einem Beruf mit sehr hoher quantitativer Arbeitsbelastung, die mit einem hohen Risiko einhergeht, dass die Pflegepersonen sich ausgebrannt fühlen, mehr Krankentage verzeichnen und eher den Beruf verlassen möchten als andere Berufsgruppen (Schmucker, 2020; Simon et al., 2005).

Die ohnehin prekäre Situation des Fachkräftemangels und die geringe Attraktivität des Pflegeberufs kann aufgrund dessen seit der Pandemie als verschärft angesehen werden. Ob die Bemühungen der Bundesregierung (bspw. durch das Pflegepersonal-Stärkungsgesetz) dieses Dilemma lösen können, scheint gerade jetzt unsicher. Weitgreifende Maßnahmen, die die Arbeitsbedingungen, das Einkommen oder die Arbeitszeiten der Pflege adressieren, sollten schnell Gegenstand der politischen Entscheidungen sein.

Pflegekräfte sind durch den regelmäßigen Umgang mit u.a. Körperflüssigkeiten einem hohen Infektionsrisiko ausgesetzt. $\mathrm{Zu}$ diesem tatsächlichen Risiko kommt die wahrgenommene Gefährdung (Simon et al., 2005). Dies ist angesichts der Tatsache, dass über die Hälfte der Pflegedienste über einen Mangel an Schutzausrüstung und ein Drittel auch über einen Mangel an Flächendesinfektionsmittel berichten, ein nicht zu vernachlässigendes Thema. Auch wenn sich die Situation fehlender Materialien seit 
Beginn der Pandemie entspannt hat, bleibt festzuhalten, dass die Versorgung zumindest zeitweise nicht sichergestellt und der Schutz für Pflegekräfte und Pflegebedürftige gefährdet war.

\section{Auswirkungen auf Pflegebedürftige}

Die Versorgungsbereiche der Pflege, Betreuung und Hauswirtschaft deckt die gesetzliche Pflegeversicherung in vielen Fällen nur teilweise ab. Um dem Versorgungsbedarf dennoch gerecht zu werden, greifen laut Petermann et al. (2020) geschätzt 250000 deutsche Haushalten auf 24-Stunden-Betreuungskräfte - sogenannte Live-ins, die vor allem aus dem osteuropäischen Ausland stammen zurück. Hielscher, Kirchen-Peters \& Nock (2017) schätzen, dass etwa jeder zwölfte Pflegehaushalt auf Live-ins zurückgreift. Da diese Hilfskräfte jedoch in $90 \%$ der Fälle nicht sozialversicherungspflichtig angestellt sind, bleibt die tatsächliche Anzahl unklar, da hierzu keine Erkenntnisse vorliegen (Deutscher Bundestag, 2019). Hielscher et al. (2017) geben weiter an, dass in ihrer Untersuchung in $11 \%$ der befragten Haushalte ein / eine Live-In beschäftigt war. In der hier vorliegenden Stichprobe waren es hingegen $5 \%$, allerdings wurde die Befragung von Hielscher und Kollegen (2017) direkt an die Pflegehaushalte gerichtet, die sicher genauere Auskünfte geben konnten, als die hier befragten ambulanten Pflegedienste.

Wer die Versorgungszeiten durch Live-ins bei den Pflegebedürftigen kompensiert, bei denen diese Leistung durch die Pandemie und damit verbundenen Einreiseverboten weggebrochen ist, bleibt angesichts der berichteten Einbrüche der Umsätze in Pflegediensten unklar. Zudem zeigen die Ergebnisse der vorliegenden Studie, dass auch teilstationäre Leistungen (wie etwa Tagespflegeeinrichtungen) massiv weggebrochen sind. Dies in Kombination mit dem beschriebenen Leistungseinbruch aus Sicht der Pflegedienste zeichnet ein besorgniserregendes Bild der Versorgung von Menschen mit Pflegebedarf während der Pandemie, das sich auch mit der Einschätzung aus den Pflegediensten deckt, dass fast die Hälfte aller Pflegedienste $(45,8 \%)$ die Versorgung von Pflegebedürftigen während der Pandemie als gefährdet/instabil oder sogar nicht sichergestellt einschätzt.

Für Gäste der teilstationären Einrichtungen und deren Angehörige führen Schließungen und die berichteten Kontaktreduzierungen $\mathrm{zu}$ erheblichen Konsequenzen für die Aufrechterhaltung von Betreuung und Versorgung - insbesondere vor dem Hintergrund, dass fast jede sechste Einrichtung auf die Versorgung von Menschen mit Demenz spezialisiert ist. Diese besonders vulnerable Gruppe von Gästen, die aufgrund ihrer kognitiven Einschränkungen nur schwer mit Veränderungen im Tagesablauf umgehen kann, ist aufgrund der benannten Einschränkungen/Schließungen besonders betroffen. Hierbei ist insbesondere der Gesichtspunkt sozialer Teilhabe hervorzuheben, der einen wichtigen Baustein im Konzept sozialer Gesundheit darstellt (Dröes et al., 2017; Huber et al., 2011) und für Men- schen mit Demenz in aller Regel nur schwer durch andere Formen der Kommunikation und Interaktion ersetzt werden kann. Der Wegfall von Versorgungsangeboten wie z.B. der Tagespflege kann hier Versorgungssicherheit gefährden. Für versorgende Angehörige entstehen große Herausforderungen, die Versorgung von Menschen mit Demenz in Zeiten der Pandemie sicherzustellen und gleichzeitig eigene Anforderungen auch in Bezug auf Beruf und / oder Kinderbetreuung auszubalancieren (Köhler, Hochgraeber \& Pinkert, 2020).

\section{Forderungen an die Politik}

Aus Sicht der befragten Dienste ergeben sich vor allem finanzielle Wünsche in Bezug auf eine Honorierung der Mitarbeitenden bzw. zur Anreizschaffung für Berufsrückkehrer. Dies ist umso nachvollziehbarer, da vor allem ambulante Pflegedienste bereits vor der Pandemie an einer Personalunterdeckung litten (Büscher et al., 2020) und fürchten, dass sich dies durch die beschlossenen Maßnahmen im Rahmen des Pflegepersonal-Stärkungsgesetz aufgrund einer Abwanderung von Pflegekräften aus der ambulanten in die stationäre oder klinische Versorgung zukünftig noch weiter verstärkt (Block, 2018; Millich, 2018). Der Wunsch nach einer vollen Kostenübernahme von Sachmitteln lässt sich sowohl unter finanziellen $\mathrm{Ge}$ sichtspunkten als auch durch die erfahrene Mangelsituation zu Beginn der Pandemie erklären. Durch die unvermeidbare körperliche Nähe in Pflegesituationen erhöht sich zudem die Gefahr einer raschen Ausbreitung der SARS-CoV-2-Virus innerhalb von Mitarbeitenden aber auch Pflegebedürftigen (Kimball et al., 2020; Kunz \& Minder, 2020; Lai et al., 2020), sodass insbesondere auch die Forderung nach ausreichenden Schutzmaterialien und einer systematischen Testung von Mitarbeitenden im Fokus steht. Diese Forderung an die Politik steht in Einklang mit den Vorschlägen zum Schutz und zur Sicherung der häuslichen Pflege in der COVID-19-Pandemie der BAGSO (Bundesarbeitsgemeinschaft der Seniorenorganisationen e.V.) und des Vereins „wir pflegen e.V.“ (bagso \& wir pflegen!, 2020).

Während die Unterstützung insbesondere durch Trägerverbände, Berufsverbände und das RKI als überwiegend positiv bewertet wurde, fühlten sich die Dienste weniger gut durch die Gesundheitsämter, BMG und Kranken- und Pflegekassen unterstützt. In Erwartung eines längeren Fortdauerns der Pandemie erscheint es daher auch sinnvoll, Maßnahmen und Unterstützungsangebote für die ambulante Pflege bei den entsprechenden Institutionen zu etablieren.

In Bezug auf die Situation der Pflegebedürftigen sind ebenfalls Maßnahmen zu erarbeiten. Neben der möglichen Wiederaufnahme von entlastenden Angeboten wie teilstationären Einrichtungen ist aber auch eine Unterstützung der pflegenden Angehörigen ratsam. Hierzu könnten beispielsweise besondere Abwesenheits- oder Heimarbeitsregelungen für berufstätige pflegende Angehörige 
zählen, die zugleich eine Reduzierung der Kontakte der Pflegebedürftigen mit sich bringen könnten.

\section{Limitationen}

Die Stichprobe der präsentierten Studie basiert auf einer Einladung zur Teilnahme an einen opportunistischen EMail-Verteiler von etwa 12000 ambulanten Pflegediensten und teilstationären Pflegeeinrichtungen. Mit einem Rücklauf von 7,3\% der Pflegedienste bzw. 3,5\% der teilstationären Einrichtungen ist die Rücklaufquote als gering einzustufen. Auf Grund des umfangreichen Verteilers konnten jedoch Antworten von einer hohen Anzahl von Pflegediensten/Einrichtungen erlangt werden. Dennoch besteht die Gefahr einer Selbstselektion und damit die Möglichkeit, dass die dargestellten Ergebnisse nicht repräsentativ für ambulante Pflegedienste und teilstationäre Einrichtungen in ganz Deutschland sind. Ein Hinweis hierfür zeigt sich darin, dass die teilnehmenden Dienste im Hinblick auf die Anzahl der Klient_innen größer sind als in der Pflegestatistik berichtet - sich allerdings aufgrund der fehlenden Differenzierung nach SGB V / XI / XIILeistungen auch nur eingeschränkt vergleichen lässt. Möglicherweise haben hier vorrangig Dienste teilgenommen, die hierfür ausreichende personelle Ressourcen aufweisen und sich somit auch im Falle der Pandemie in höherem Maße engagieren können.

Darüber hinaus wurden in dieser Befragung die Vertreter_innen der Pflegedienste stellenweise zur individuellen Situation der versorgten Klient_innen befragt, ohne dass davon auszugehen ist, dass für alle diese Angaben eine strukturierte und abrufbare Dokumentation vorlag.

\section{Schlussfolgerungen}

Die vorliegenden Ergebnisse zeigen, dass ambulant versorgten Pflegebedürftigen und damit insbesondere dem Setting ambulanter Pflegedienste in der vorliegenden Pandemie - und auch mit Blick auf eine mögliche zweite Welle - eine erhöhte Aufmerksamkeit zukommen sollte. Richtet sich der Blick von Politik und Öffentlichkeit derzeit vornehmlich auf Kapazitäten einer klinischen und intensivmedizinischen Versorgung und geraten stationäre Langzeitpflegeeinrichtungen noch in den Fokus, weil dort sehr schnell Hotspots der Erkrankung mit vielen hochbetagten und multimorbiden Menschen auf engem Raum entstehen können, so verliert sich die Aufmerksamkeit in ambulanten Versorgungssituationen, obwohl dort ebenfalls sehr große und sehr vulnerable Gruppen von Personen versorgt werden. Eine gesicherte ambulante Versorgung Pflegebedürftiger hat zudem auch für viele Angehörige eine hohe Relevanz, um weiter ihren beruflichen Tätigkeiten nachgehen zu können. Hier findet sich somit ein Dilemma, da entlastende Angebote wie Tagespflege zwar einen wichtigen Teil in der Versorgung darstellen, als soziale Aktivität jedoch auch das Risiko einer stärkeren Ausbreitung der Pandemie bergen.

Wichtige konkrete Forderungen in Bezug auf eine qualitativ hochwertige ambulante Versorgung auch in der Ausnahmesituation einer Pandemie sind daher eine umfassende und sehr schnelle Testung von Verdachtsfällen sowohl bei Klient_innen als auch bei Mitarbeitenden und eine ausreichende Bevorratung mit Schutzmaterialien. Auch die Erarbeitung bundeseinheitlicher Pandemiepläne gehören zu den Lehren, die aus der vorliegenden Situation gezogen werden sollten. Hierbei ist im Blick zu behalten, dass notwendige Schutzmaßnahmen wie etwa die verringerte Inanspruchnahme von Leistungen, reduzierte Kontakte und soziale Distanzierung gerade im ambulanten Bereich zu prekären Versorgungssituationen führen können.

\section{Literatur}

bagso \& wir pflegen! (2020). Vorschläge zum Schutz und zur Sicherung der häuslichen Pflege in der Corona-Pandemie. Verfügbar unter https://www.bagso.de/fileadmin/user_upload/bagso/01_ News/Aktuelles/2020/Massnahmen_zur_Corona-Soforthilfe_ fuer_die_haeusliche_Pflege.pdf [24.06.2020].

Begerow, A. \& Gaidys, U. (2020). COVID-19 Pflege Studie. Erfahrungen von Pflegenden während der Pandemie - erste Teilergebnisse. Pflegewissenschaft (Sonderausgabe: Die Corona-Pandemie), $85-86$.

Block, S. (2018). Kritik am neuen Pflegepersonal-Stärkungsgesetz. Kommentar. Häusliche Pflege. Verfügbar unter http:// www.haeusliche-pflege.net/Infopool/Nachrichten/Kritik-amneuen-Pflegepersonal-Staerkungsgesetz [24.06.2020].

Büscher, A., Schröder, D., Gruber, E. M., Bäumker, M. T. \& Zeiser, S. (2020). Empfehlungen zur Entwicklung von personellen Vorgaben für ambulante Pflegeeinrichtungenim Rahmen der „Entwicklung eines wissenschaftlich fundierten Verfahrens zur einheitlichen Bemessung des Personalbedarfs in Pflegeeinrichtungen nach qualitativen und quantitativen Maßstäben gemäß \$113 c SGB XI“. Abschlussbericht. Osnabrück. Verfügbar unter https://www. gs-qsa-pflege.de/wp-content/uploads/2020/02/Anlagenbandzum-2.-Zwischenbericht.pdf [24.06.2020].

Deutscher Bundestag (2019). Antwort der Bundesregierung auf die Kleine Anfrage der Abgeordneten Pia Zimmermann, Susanne Ferschl, Matthias W. Birkwald, weiterer Abgeordneter und der Fraktion DIE LINKE. - Drucksache 19/6392 - Arbeitsbedingungen von im Haushalt lebenden Pflegekräften Köln. Verfügbar unter http://dip21.bundestag.de/dip21/btd/19/067/1906792.pdf [23.06.2020].

Dibelius, O. \& Piechotta-Henze, G. (2020). Care Work zu Hause. Die Versorgung von älteren Menschen durch osteuropäische Arbeitsmigrant_innen. Pflegewissenschaft (Sonderausgabe: Die Corona-Pandemie), $85-86$.

Hielscher, V., Kirchen-Peters, S. \& Nock, L. (2017). Pflege in den eigenen Vier Wänden: Zeitaufwand und Kosten. Pflegebedürftige und ihre Angehörigen geben Auskunft. Stuttgart. Verfügbar unter https: //www.boeckler.de/pdf/p_study_hbs_363.pdf [17.08.2020].

Ing, A. J., Cocks, C. \& Green, J. P. (2020). COVID-19: in the footsteps of Ernest Shackleton. Thorax, 75 (8), 693 - 694.

Kimball, A., Hatfield, K. M., Arons, M., James, A., Taylor, J., Spicer, K. et al. (2020). Asymptomatic and Presymptomatic SARS-CoV-2 Infections in Residents of a Long-Term Care Skilled Nursing Facility - King County, Washington, March 2020. Morbidity and Mortality Weekly Report, 69 (13), 377 - 381.

Köhler, K., Dreyer, J., Hochgraeber, I., Pinkert, C. \& Holle, B. (2020). Gefährdet die Covid-19-Pandemie die Stabilität häuslicher Versorgung von pflegebedürftigen Menschen mit einer Demenz? - 
eine Refelktion. Pflegewissenschaft (Sonderausgabe: Die Corona-Pandemie), $87-90$.

Kunz, R. \& Minder, M. (2020). COVID-19 pandemic: palliative care for elderly and frail patients at home and in residential and nursing homes. Swiss Medical Weekly, 150, w20235.

Lai, C. C., Wang, J. H., Ko, W. C., Yen, M. Y., Lu, M. C., Lee, C. M. et al. (2020). COVID-19 in long-term care facilities: An upcoming threat that cannot be ignored. Journal of Microbiology, Immunology and Infection, 53 (3), $444-446$

Millich, N. (2018). Pflegepersonal-Stärkungsgesetz. Reaktionen zeigen: Es bleibt noch viel zu tun. BibliomedPflege. Verfügbar unter https://www.bibliomed-pflege.de/news/36823-reaktionenzeigen-es-bleibt-noch-viel-zu-tun [24.06.2020].

Ministerium für Arbeit, Gesundheit und Soziales des Landes Nordrhein-Westfalen (2020). Verordnung zum Schutz vor Neuinfizierungen mit dem Coronavirus SARS-CoV-2 2 im Bereich der Betreuungsinfrastruktur (Coronabetreuungsverordnung-CoronaBetrVO). In der ab dem 7. Mai 2020 gültigen Fassung. Verfügbar unter https://www.mags.nrw/sites/default/files/asset/document/ 2020-04-16_neufassung_coronabetrvo_ab_20.04.2020.pdf [24.06.2020].

Petermann, A., Jolly, G. \& Schrader, K. (2020). Fairness und Autonomie in der Betreuung in häuslicher Gemeinschaft - Ergebnisse einer empirischen Studie. In B. Städtler-Mach \& H. Ignatzi (Hrsg.), Grauer Markt Pflege. 24-Stunden-Unterstützung durch osteuropäische Betreuungskräfte. (S.99-122). Göttingen: Vandenhoeck \& Ruprecht.

Questback GmbH. (2019). EFS Survey, Version Fall 2019. Köln: Questback GmbH. Verfügbar unter https://www.questback. com/de/online-befragungstool/ [17.08.2020].

RKI (2020a). COVID-19-Lagebericht vom 05.05.2020. Verfügbar unter https://www.rki.de/DE/Content/InfAZ/N/Neuartiges_Corona virus/Situationsberichte/2020-05-05-de.pdf?_blob=publicationFile [12.07.2020].

RKI (2020b). SARS-CoV-2 Steckbrief zur Coronavirus-Krankheit-2019 (COVID-19). Verfügbar unter https://www.rki.de/DE/ Content/InfAZ/N/Neuartiges_Coronavirus/Steckbrief. html\#doc13776792bodyText8 [24.06.2020].

RKI (2020c). Täglicher Lagebericht des RKI zur Coronavirus-Krankheit-2019 (COVID-19). 05.05.2020 Aktualisierter Stand für Deutschland. Verfügbar unter https://www.rki.de/DE/Content/ InfAZ/N/Neuartiges_Coronavirus/Situationsberichte/202005-05-de.pdf?__blob=publicationFile [24.06.2020].

Rothgang, H., Domhoff, D., Friedrich, A. C., Heinze, F., Preuß, B., Schmidt, A. et al. (2020). Pflege in Zeiten von Corona - Erebnisse einer bundesweiten Querschnittsbefragung vollstationärer Pflegeeinrichtungen in Deutschland. Pflege, 33 (5), 265 - 275.

Schmucker, R. (2020). Arbeitsbedingungen in Pflegeberufen. In K. Jacobs, A. Kuhlmey, S. Greß, J. Klauber \& A. Schwinger (Hrsg.), Pflege-Report 2019 (S. 49 -60). Berlin, Heidelberg: Springer.

Simon, M., Tackenberg, P., Hasselhorn, M. H., Kümmerling, A., Büscher, A. \& Müller, B. H. (2005). Auswertung der ersten Befragung der NEXT-Studie in Deutschland. Wuppertal. Verfügbar unter https://www.researchgate.net/profile/Michael_Simon/publication /325908204_Auswertung_der_ersten_Befragung_der_NEXTStudie_in_Deutschland/links/5b2bf38fa6fdcc8506bc6c3d/ Auswertung-der-ersten-Befragung-der-NEXT-Studie-inDeutschland.pdf [24.06.2020].

Statistisches Bundesamt (2018). Pflegestatistik 2017. Pflege im Rahmen der Pflegeversicherung Deutschlandergebnisse. Verfügbar unter https://www.destatis.de/DE/Themen/GesellschaftUmwelt/Gesundheit/Pflege/Publikationen/Downloads-Pflege/ pflege-deutschlandergebnisse-5224001179004.pdf?_blob=publicationFile [24.06.2020].
Stobbe, M. (2020). More evidence indicates healthy people can spread virus. AP News. Verfügbar unter https://apnews.com/5c 4992645fee551994325093858c14a4 [24.06.2020].

UN (2020). Policy Brief: The Impact of COVID-19 on older persons. Verfügbar unter https://unsdg.un.org/sites/default/files/202005/Policy-Brief-The-Impact-of-COVID-19-on-Older-Persons.pdf [24.06.2020].

\section{Historie}

Manuskripteingang: 01.06.2020

Manuskript angenommen: 29.08.2020

\section{Autorenschaft}

Substanzieller Beitrag zu Konzeption oder Design der Arbeit: KW, AS, BP, KS, AF, DD, CS, HR

Substanzieller Beitrag zur Erfassung, Analyse oder Interpretation der Daten: KW, AS, BP, FH, KS, AF, DD, CS, HR

Manuskripterstellung: KW, AS, BP, FH, KS, AF, DD, HR

Einschlägige kritische Überarbeitung des Manuskripts: KW, HR, $\mathrm{KS}, \mathrm{DD}$

Genehmigung der letzten Version des Manuskripts: KW, AS, BP, $\mathrm{FH}, \mathrm{KS}, \mathrm{AF}, \mathrm{DD}, \mathrm{CS}, \mathrm{HR}$

Übernahme der Verantwortung für das gesamte Manuskript: $\mathrm{KW}, \mathrm{AS}, \mathrm{BP}, \mathrm{FH}, \mathrm{KS}, \mathrm{AF}, \mathrm{DD}, \mathrm{CS}, \mathrm{HR}$

\section{ORCID}

Karin Wolf-Ostermann

(D) https://orcid.org/0000-0001-8513-3125

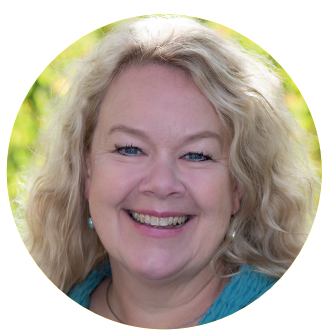

Prof. Dr. Karin Wolf-Ostermann

Health Sciences Bremen

Universität Bremen

PF 330440

28334 Bremen

Deutschland

Was war die größte Herausforderung bei Ihrer Studie?

Pflegedienste und -einrichtungen trotz der hochbelastenden Situation zur Mitarbeit zu gewinnen.

\section{Was wünschen Sie sich bezüglich der Thematik für die Zukunft?}

Ambulante und teilstationäre Langzeitpflege muss nachhaltig verbessert und die Vermeidung prekärer Versorgungssituationen trotz Pandemie sichergestellt werden.

\section{Was empfehlen Sie zum Weiterlesen/Vertiefen?}

Wir verweisen auf Rothgang et al. (2020, in diesem Heft) sowie aktuelle qualitätsgesicherte Internetressourcen (z. B. https:// (tccovid.org/). 